\title{
Controlling Spatiotemporal Chaos in a Realistic El Niño Prediction Model
}

\section{Citation}

Tziperman, Eli, Harvey Scher, Stephen E. Zebiak, and Mark A. Cane. 1997. Controlling spatiotemporal chaos in a realistic El Nino prediction model. Physical Review Letters 79(6): 1034-1037.

\section{Published Version}

http://dx.doi.org/10.1103/PhysRevLett.79.1034

\section{Permanent link}

http://nrs.harvard.edu/urn-3:HUL.InstRepos:3425922

\section{Terms of Use}

This article was downloaded from Harvard University's DASH repository, and is made available under the terms and conditions applicable to Other Posted Material, as set forth at http:// nrs.harvard.edu/urn-3:HUL.InstRepos:dash.current.terms-of-use\#LAA

\section{Share Your Story}

The Harvard community has made this article openly available.

Please share how this access benefits you. Submit a story.

\section{Accessibility}




\title{
Controlling Spatiotemporal Chaos in a Realistic EI Niño Prediction Model
}

\author{
Eli Tziperman and Harvey Scher \\ Environmental Sciences, The Weizmann Institute of Science, Rehovot 76100, Israel \\ Stephen E. Zebiak and Mark A. Cane \\ Lamont-Doherty Earth Observatory, Columbia University, Palisades, New York 10964
}

(Received 28 March 1997)

\begin{abstract}
A method for controlling low-order chaotic behavior of continuous spatiotemporal systems is developed and demonstrated in a complex, realistic 3D partial differential equation model that is used successfully for predicting El Niño events in the equatorial Pacific. An unstable periodic orbit that involves a full-domain oscillation is stabilized using a feedback control applied to a single degree of freedom at a carefully chosen single "choke point" in space. A general criterion is presented for determining the optimal points in reconstructed delay-coordinate phase space at which to apply the feedback control. [S0031-9007(97)03780-0]
\end{abstract}

PACS numbers: $05.45 .+\mathrm{b}, 47.52 .+\mathrm{j}, 92.10 . \mathrm{Gk}$

There has been significant interest in recent years in the control of low-order chaotic dynamical systems using small systematic perturbations that lead to the stabilization of unstable periodic orbits (UPO) [1,2]. Controlling large- or infinite-dimensional systems, however, such as spatiotemporal systems that are governed by partial differential equations, is still in its infancy [3-10]. We present here a new approach to the control of spatiotemporal systems that are continuous in both space and time. The proposed method is successfully used to control a highly complex model, composed of a 3D spatiotemporal system of partial differential equations (PDE's), which simulates the El Niño phenomenon in the equatorial Pacific ocean and atmosphere. Spatiotemporal systems often do not have a system-wide adjustable parameter that can be used for an Ott-Grebogi-York (OGY) $[1,10]$ control. Our approach does not require the existence of such a global parameter; rather it is based on applying OGY-like feedback perturbations to a single degree of freedom of the system [11], at a single point in space. The spatiotemporal system successfully controlled here seems to be significantly more complicated than the previously controlled discrete systems of coupled chaotic elements [4,6], or relatively simple or isotropic 1D or 2D systems of PDE's [3,5,7,8]. Often, the precise dynamics responsible for the chaotic behavior of systems of such complexity, whether they are models or experimental systems, is not known. We therefore use delay-coordinate phase space reconstruction for deriving and applying the control law, thus not requiring a knowledge of the detailed dynamics of the system [12]. Our approach controls the continuous spatiotemporal system using a continuous reconstruction of the UPO in phase space; we consequently find that not every point on the continuous UPO may be used to apply the control, and present a novel general criterion for determining the controllability of phase space points along a given UPO.

While we obviously do not propose here the control of actual El Niño events, we do show that chaos control in a realistic El Niño model can contribute to the understanding of El Niño's dynamics. El Niño events involve a widespread warming of the equatorial Pacific Ocean surface water (Fig. 1). They occur irregularly in time, about every 2-6 years, dramatically affect worldwide weather, and have important social and economic implications [13]. Recent theories [14,15] attribute El Niño's irregularity to a low order chaos. An important lesson of the present work is that the control of complex spatiotemporal systems may not be achieved using an "out-of-the-box" algorithm, but requires a good understanding of the relevant dynamics. We thus briefly describe now the model complexity and the relevant aspects of El Niño dynamics before proceeding to the control algorithm. The El

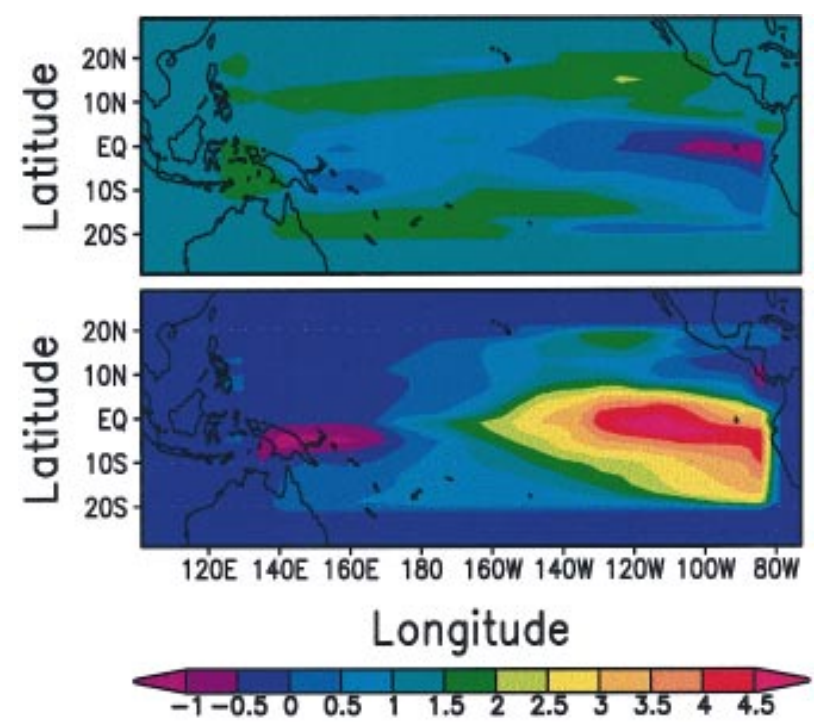

FIG. 1(color). The deviation of the sea surface temperature $\left({ }^{\circ} \mathrm{C}\right)$ from the long-term mean during a peak of a model $\mathrm{El}$ Niño (warming) event (lower panel) and during the peak of a La Nina (cooling) event (upper panel) which typically occurs between El Niño events. 
Niño model used here (described in detail in [16]) has proven quite successful in predicting El Niño events up to one and a half years in advance [17]. It is based on sets of nonlinear partial differential equations for the ocean and for the atmosphere, with specified coupling between them. The equations are written for the deviations from the observed spatially variable long-term mean state of the equatorial Pacific. This mean state may be seasonally varying [16], and in the model version used here is set to the time-independent mean July state. In the model equations $t$ is the time, $(x, y, z)$ are the (east, north, up) coordinates, $(u, v, w)$ the corresponding ocean water velocities, $\left(u_{a}, v_{a}\right)$ the atmospheric wind velocity in the (east, north) directions, $\mathbf{v}=(u, v), \mathbf{v}_{a}=\left(u_{a}, v_{a}\right)$, and $\nabla=\left(\partial_{x}, \partial_{y}\right)$; the total depth of warm surface waters in the model, also known as the "thermocline" depth, is $h(x, y, t) ; T(x, y, t)$ is the sea surface temperature (SST). $\overline{\mathbf{v}}, \bar{T}$, and $\bar{w}$ are the spatially variable specified observed long-term July mean fields; $\beta=d f / d y$ is the gradient of the Coriolis parameter; and the terms with $r$ and $\alpha$ represent dissipation processes. $H$ is a mean thermocline depth, and $g^{\prime}$ denotes gravity acceleration. The model oceanic currents are driven by the atmospheric wind stress, $\left(\tau^{(x)}, \tau^{(y)}\right)$, which is quadratically related to the wind velocity $\left(u_{a}, v_{a}\right)$. The atmospheric winds are driven by a heating, $Q\left[T, \nabla \mathbf{v}_{a}\right]$ which is a nonlinear function of the SST and of the atmospheric wind divergence. The model is composed of four sets of PDE's. The first set is of the ocean momentum and mass conservation equations,

$$
\begin{aligned}
u_{t}-\beta y v & =-g^{\prime} h_{x}+\tau^{(x)}\left[u_{a}, v_{a}\right]-r u \\
\beta y u & =-g^{\prime} h_{y}+\tau^{(y)}\left[u_{a}, v_{a}\right]-r v \\
h_{t}+H \nabla \mathbf{v} & =-r h .
\end{aligned}
$$

A second similar set of PDE's is used to simulate the vertical velocity shear in the ocean and a third effectively 2D set of PDE's models the momentum and mass balances of the atmosphere. Finally, the SST is determined by a nonlinear advection-dissipation equation roughly of the form

$$
\begin{aligned}
T_{t}+\overline{\mathbf{v}} \nabla T+\mathbf{v} \nabla(\bar{T}+T)+w \bar{T}_{z}+ \\
(\bar{w}+w) T_{z}=-\alpha T .
\end{aligned}
$$

The model's finite-difference discretization is based on many thousands of grid point variables. The model solution is aperiodic and involves unstable interactions between the ocean and the atmosphere, which are manifested through multiple spatial and temporal scales of all model fields. Yet, the previously demonstrated low-order temporally chaotic behavior of this model [15] makes it a perfect candidate for testing chaos-control ideas in a complex, high dimensional, spatially extended system. The challenge, of course, is to control an UPO that represents the full-domain oscillation of the El Niño cycle without applying the chaoscontrol corrections at many spatial locations.

One of the main keys to controlling chaos in a complex spatiotemporal system using the approach suggested here, is the careful identification of the correct spatial point and degree of freedom (model variable or physical quantity in an experimental system) to which control corrections are applied, based on an understanding of the system's dynamics. The variation of the Coriolis parameter with latitude $[\beta y$ in (1)] results in the equator being a waveguide for trapped ocean wave modes which have the form $H_{n}(y / \ell) \exp \left(-\frac{1}{2} y^{2} / \ell^{2}\right) \exp i\left(k x-\sigma_{n} t\right)$, with $H_{n}$ being the Hermite polynomial of order $n$, and where $\ell=3.2$ degrees latitude. The mode $n=0$ is eastward propagating and is known as an equatorial Kelvin wave, and the $n>1$ modes are westward propagating, off equatorial, Rossby modes. These equatorial ocean Kelvin and Rossby waves play a central role in El Niño's dynamics [13]. To see how these modes may be used to control the chaotic behavior in this model, let us briefly examine the mechanism of the El Niño cycle, known as the "delayed oscillator" mechanism [18].

Consider a weakening of the easterly winds $\left(u_{a}\right)$ in the central equatorial Pacific, which causes warm water to shift from higher latitudes toward the equator, creating a warm perturbation at the equator and cold perturbations off the equator. The resulting deepening thermocline (larger $h$ ) perturbation at the equator propagates eastward as a warm Kelvin wave, reaching the eastern boundary after about 1 month. Upon reaching the eastern boundary, the thermocline deepening signal induces a warm SST perturbation which reduces the east-west SST gradient, and thus further weakens the easterly winds above the equator. The weakened easterly winds amplify the deepening waves, creating a positive feedback (i.e., a coupled ocean-atmosphere instability) that leads to a rapid warming in the eastern equatorial Pacific Ocean, starting an El Niño event. Meanwhile, the initial cold SST perturbations off the equator in the central Pacific excite upwelling (i.e., smaller $h$ ) perturbations which travel westward as Rossby waves, and are reflected at the western boundary as cold equatorial Kelvin waves. Amplified again by the atmospheric feedback, these cold Kelvin waves reach the Eastern Pacific, delayed by about 6 months after the original wind perturbation, and terminate the El Niño event.

The western boundary of the ocean at the equator is a "choke point" which affects the entire tropical Pacific through the reflection of the Rossby waves into Kelvin waves [18]. We therefore chose to control the entire equatorial Pacific model ocean and atmosphere by applying small perturbations to the oceanic model fields at the western boundary $\left(x=x_{w}\right)$ of the Pacific Ocean. The applied control perturbations have the $y$ structure of the Kelvin mode. Our control variable is thus the Kelvin mode amplitude at the western boundary, $K_{w}(t)$, and is a single degree of freedom out of thousands which exist in the model. Because the Kelvin mode amplitude decays like $\exp \left(-\frac{1}{2} y^{2} / \ell^{2}\right)$ away from the equator, the control correction directly affects the oceanic model fields only in a very small region near the equator, at the western boundary. 
As the first step in applying our method to the control of chaos in this model, we determine its UPO's in an $N$ dimensional delay-coordinate phase-space reconstructed from the Kelvin wave amplitude at the western boundary $\mathbf{X}(t)=\left\{X_{i}, i=1, \ldots, N\right\}=\left\{K_{w}[t-\right.$ $\left.(N-1) \tau], \ldots, K_{w}(t-\tau), K_{w}(t)\right\}^{T}$. For a given period $p$ we search for phase space points $X(t)$ that return to the same neighborhood after a period $p$, so that $\| \mathbf{X}(t)-$ $\mathbf{X}(t-p) \|<\epsilon$ for some small $\epsilon$. Using $\tau=1$ year, and plotting the number of such close pairs as a function of $p$, the UPO's show up as peaks [Fig. 2(a)]. Two of the UPO's are shown in Figs. 2(c) and 2(d). The first UPO corresponds to a relatively weak El Niño event every 4.3 years, while the second corresponds to a strong event followed by a very weak one, repeating every 7.83 years.

Next, an $N \times N$ linear map, $M$, is least-square-fitted to the model dynamics over a small neighborhood in phase space near a point that is located along the controlled unstable periodic orbit, and that serves as the control point in phase space, by minimizing $J(M)=\sum_{t} \| \mathbf{X}(t)-$ $M \mathbf{X}(t-p) \|^{2}$.

The feedback control correction is now calculated so that when the system approaches the control point in phase space, the correction brings the phase space trajectory toward the stable manifold of the UPO. The
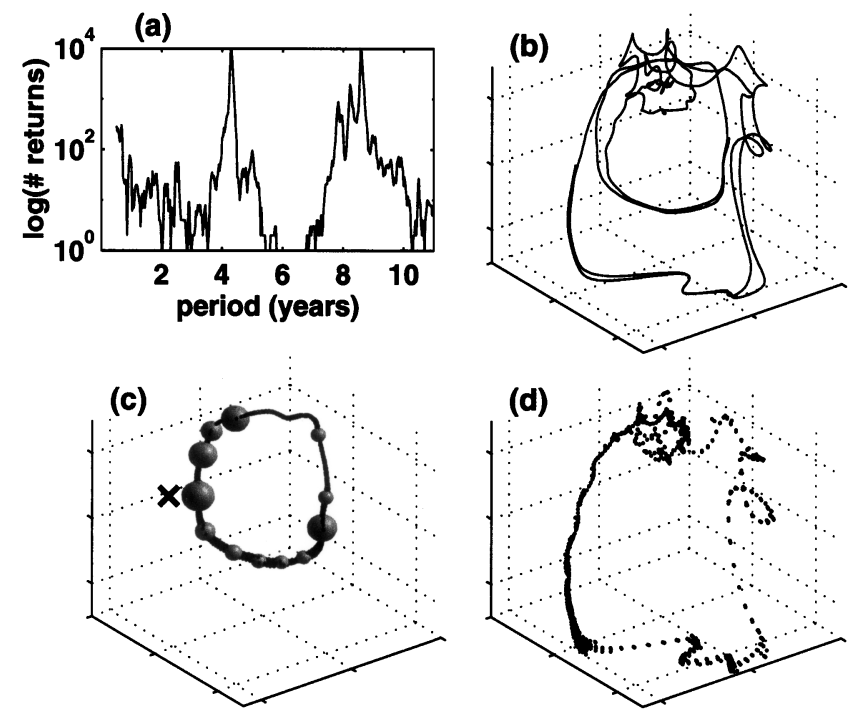

FIG. 2. (a) Log of number of near returns as a function of the period $p$. Each peak corresponds to an UPO, and the peak at $p=4.3$ years is the one stabilized here. (b) A segment of phase space trajectory during a typical standard run, showing the trajectory switching between the two UPO's of panels (c) and (d). The three axes are $\left[K_{w}(t-2 \tau), K_{w}(t-\tau), K_{w}(t)\right]$. (c) A $3 \mathrm{D}$ reconstructed delay coordinate phase space plot of the near returns forming the $p=4.3$ years UPO that is stabilized in the controlled run. Larger balls along the UPO denote smaller $G_{N N}$ and thus more controllable phase space points (see text). The most controllable phase space point, with the smallest $G_{N N}$, where the control correction is actually applied, is marked by " $\times$ ". (d) A UPO with a period of $p=7.83$ years. model evolution will then bring the trajectory of the control variable toward the UPO itself [1]. If our choice of a choke point in space is appropriate, the entire 3D model solution will follow the control variable and settle on the UPO as well. Suppose that the linear map $M$, evaluated at the control point, has $N_{s}$ stable eigenvalues whose eigenvectors span the stable manifold. Let $S$ be an $N \times N_{s}$ rectangular matrix composed of these $N_{s}$ stable eigenvectors. Let $\mathbf{X}(t)$ be the phase space location of the model trajectory at time $t$, defined with the origin at the control point along the controlled UPO. The phase space location in the stable manifold to which we wish to bring the model trajectory can be written as $S \mathbf{a}$ where a is some $N_{s} \times 1$ coefficient vector. The control correction can be applied only to the present time Kelvin amplitude $K_{w}(t)=X_{N}(t)$, so that the phase space trajectory can only be corrected in the direction of a unit vector $\hat{\mathbf{X}}_{N}$ along the $N$ th axis in phase space. The phase space location after the application of the control perturbation $\delta X_{N}$ is, therefore, $\mathbf{X}(t)+\hat{X}_{N} \delta X_{N}$. We are interested in the control correction $\delta X_{N}$ for which the distance of the corrected phase space location to the stable manifold, $d=$ $\left\|S \mathbf{a}-\left[\mathbf{X}(t)+\hat{\mathbf{X}}_{N} \delta X_{N}\right]\right\|$, vanishes. $\delta X_{N}$ is obtained by solving $\partial d / \partial \mathbf{a}=\mathbf{0}$ and $\partial d / \partial \delta X_{N}=0$ for the $\mathbf{a}$ and $\delta X_{N}$ which minimize $d$, finding

$$
\delta X_{N}=\left(X_{N}-\hat{\mathbf{X}}_{N}^{T} G \mathbf{X}\right) /\left(\hat{\mathbf{X}}_{N}^{T} G \hat{\mathbf{X}}_{N}-1\right),
$$

where $G=S\left(S^{T} S\right)^{-1} S^{T}$. Given a time series from any spatiotemporal system, this simple control law may always be derived without additional knowledge of the dynamics. In the present case, we used $N=3$ and found that typically there is one unstable eigenvalue of $M$ (whose value, representing the amplification over a full orbit around the UPO, typically varies around 1.5), one neutral (value close to 1 ) and one stable eigenvalue $(<1)$, so that we set $N_{s}=2$.

One of our more generally applicable results here is a procedure for choosing the phase space points along a given UPO at which control may be applied. Our control correction is always applied in the direction of $\hat{\mathbf{X}}_{N}$ in the reconstructed phase space. If, for some control point, this direction is parallel to the stable manifold, the control perturbations along $\hat{\mathbf{X}}_{N}$ cannot bring the phase space trajectory away from the unstable manifold. This uncontrollable situation can be shown to happen when $G_{N N}=\hat{\mathbf{X}}_{N}^{T} G \hat{\mathbf{X}}_{N}=1$, leading according to (3) to an infinite amplitude correction. Likewise, a smaller $G_{N N}$ implies a smaller perturbation $\delta X_{N}$ required to bring $X(t)$ to the stable manifold, and thus a better phase space point to apply the control. Figure 2(c) shows how the variation of $G_{N N}$ along the stabilized UPO may be used to choose an appropriate control point in phase space.

During the model integration, the control correction $\delta X_{N}$ is calculated using (3). The Kelvin wave amplitude at $x_{w}$ is corrected by $\delta X_{N}$ only when the model trajectory in phase space nears the control point, and only when 


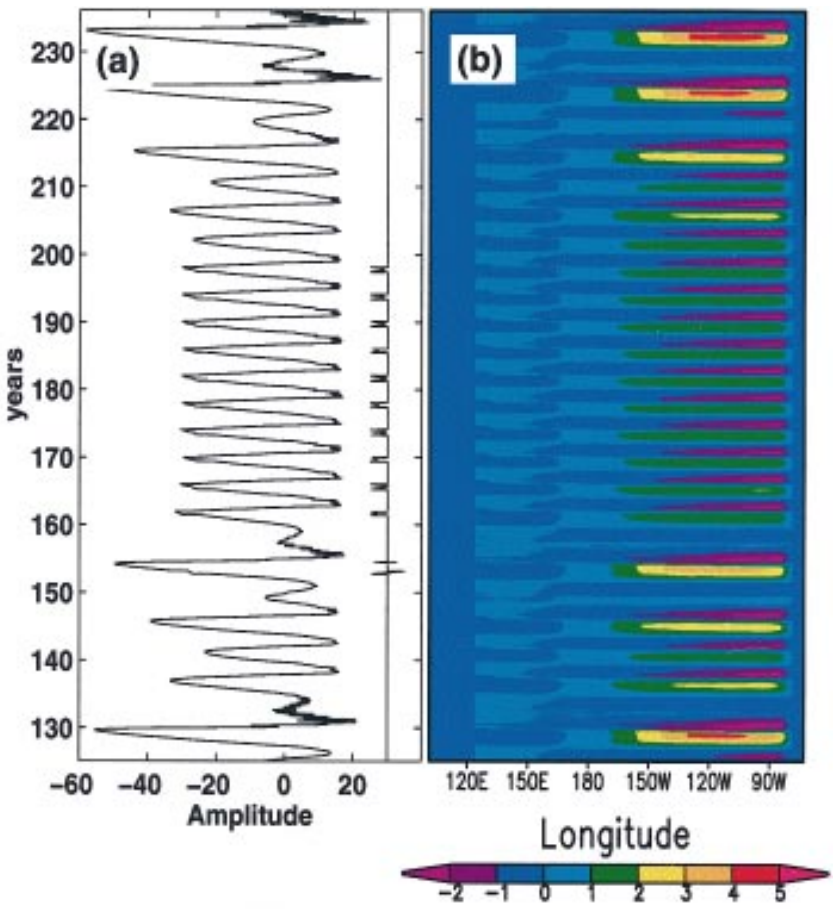

FIG. 3(color). (a) Left curve: a time series of the Kelvin wave amplitude on the western boundary of the Pacific Ocean, to which the control is applied from year 150 to 200 . Right curve: the magnitude of the applied control perturbation. (b) (color) A plot of the equatorial sea surface temperature $\left({ }^{\circ} \mathrm{C}\right.$ ) as a function of longitude and time during the same run. The controlled, periodic behavior during years 150-200 represents a full-domain oscillation of a complex spatial structure and temporal evolution.

$\delta X_{N}$ is smaller than a prespecified threshold. Figure 3 shows the model solution with and without control, demonstrating that the procedure outlined here indeed works most efficiently for this complex El Niño model.

Previous works debated whether the aperiodicity in the El Niño model used here is due to low-order chaos [15], or due to high frequency, small spatial-scale, air-sea interactions in the western Pacific [reflected in Fig. 3(a) as an intermittent noise signal at years 130-135, for example] [19]. The existence of unstable manifolds of the UPO's and the successful control of chaos in this El Niño model are a clear demonstration that the aperiodicity in this model is due to low-order chaos (whether El Niño events in the actual equatorial Pacific are aperiodic due to chaos or noise is still under debate). Each unstable spatiotemporal UPO in this system is, of course, characterized by both different temporal evolution and different spatial patterns. This leads to an especially interesting possibility that the small-scale, high-frequency "noise" in spatiotemporal systems (such as seen in the Western Pacific in this model) may be a result of low-order behavior, due to the large-scale spatial fields readjusting when jumping from one UPO to another.

The successful application of the chaos control method presented here to a complex PDE El Niño model is a clear demonstration of the robustness and potential of the method. In addition, the results presented here may also contribute to the important problem of understanding and predicting El Niño events in the equatorial Pacific.

This research was partially supported by the IsraelU.S. Binational Science Foundation.

[1] E. Ott, C. Grebogi, and J. Yorke, Phys. Rev. Lett. 64, 1196 (1990).

[2] T. Shinbrot, Adv. Phys. 44, 73-111 (1995); L. W. Ditto, M.L. Spano, and J.F. Lindner, Physica D 86, 198-211 (1995).

[3] I. Aranson, H. Levine, and L. Tsimring, Phys. Rev. Lett. 72, 2561-2564 (1994).

[4] D. Auerbach, Phys. Rev. Lett. 72, 1184 (1994).

[5] C. Lourenco, M. Hougardy, and A. Babloyantz, Phys. Rev. E 52, 1528-1532 (1995).

[6] A. Babloyantz, C. Lourenco, and J.A. Sepulchre, Physica D 86, 274-283 (1995).

[7] Y. Braiman, J.F. Lindner, and W.L. Ditto, Nature (London) 378, 465-467 (1995).

[8] G. Hu, Z. Qu, and K. He, Int. J. Bifurcation and Chaos 5, 901-936 (1995).

[9] M. Kushibe, Y. Liu, and J. Ohtsubo, Phys. Rev. E 53, 4502-4508 (1996).

[10] M. Ding, W. Yang, V. In, W. L. Ditto, M. L. Spanno, and B. Gluckman, Phys. Rev. E 53, 4334-4344 (1996).

[11] A. Garfinkel, M. L. Spano, W. L. Ditto, and J. N. Weiss, Science 257, 1230-1235 (1992).

[12] U. Dressler and G. Nitsche, Phys. Rev. Lett. 68, 1-4 (1992).

[13] S. G. Philander, El Niño, La Niña, and the Southern Oscillations (Academic Press, San Diego, CA, 1990).

[14] F-F. Jin, D. Neelin, and M. Ghil, Science 264, 70-72 (1994); P. Chang, B. Wang, T. Li, and L. Ji, Geophys. Res. Lett. 21, 2817-2820 (1994); G. K. Vallis, Science 232, 243 (1986).

[15] E. Tziperman, L. Stone, M. A. Cane, and H. Jarosh, Science 264, 72-74 (1994); E. Tziperman, M. A. Cane, and S. Zebiak, J. Atmos. Sci. 52, $293-306$ (1995).

[16] S. E. Zebiak and M. A. Cane, Mon. Wea. Rev. 115, 2262 (1987).

[17] M. A. Cane, S.E. Zebiak, and S.C. Dolan, Nature (London) 321, 827 (1986); D. Chen, S.E. Zebiak, A. J. Busalacchi, and M. A. Cane, Science 269, 1699 (1995).

[18] M. J. Suarez and P.S. Schopf, J. Atmos. Sci. 45, 3283 (1988); N. E. Graham and W. B. White, Science 240, 1293 (1988); D. S. Battisti, J. Atmos. Sci. 45, 2889 (1989).

[19] J. N. Mantua and D. S. Battisti, J. Climate 8, 2897-2927 (1995). 